\title{
Benefits of Sports Activities with FITT Principles During the Covid-19 Pandemic in a "New Normal" Life for Health
}

\author{
Sapto Adi ${ }^{*}$ \\ ${ }^{1}$ Universitas Negeri Malang \\ *Corresponding author.Email: sapto.adi.fik@um.ac.id
}

\begin{abstract}
This article aims to provide an understanding of the importance of doing sports activities during the COVID19 pandemic to maintain fitness and health. In this article, the author wants to explain that using the principle of frequency, intensity, time, and type (FITT) in sports activities will provide convenience for the community in remembering how to do sports activities independently. Likewise, the author wants to provide information about the types of sports that can be done or which are the choice during the COVID-19 pandemic at the "new normal" stage. From the results of studies in various journals, that physical activity/exercise has a positive impact on physical and mental health. The benefits of doing physical activity/exercise are basically relatively similar between those who use FITT guidelines and those who don't. However, based on the principle of FITT training, the community has a clearer direction in activities. Exercising using FITT guidelines can improve physical fitness and contribute to the management of some lifestyle-related diseases such as diabetes, hypertension, dyslipidemia, obesity, and metabolic syndrome. In addition, from the aspect of mental health is able to develop self-efficacy and other affective attributes that can motivate lifelong compliance.
\end{abstract}

Keywords - physical activity/exercise, FITT principle, "New normal", health.

\section{INTRODUCTION}

\section{Moving Basic Human Needs}

Moving is one of the basic human needs to sustain life. Humans move to work to find food, care for themselves, worship, move to achieve the desired goals, and fill spare time according to their hobbies. At certain ages, especially in old age, the organs of the human body experience a decline in functions such as muscle strength, bones, cardiovascular function, and mental aspects. Therefore, so that the decline in function is not rapid, physical activities/sports can be carried out routinely, planned, and systematically by using the FITT principle.

\section{Benefits of Exercising}

\section{a. Health}

Sports activities are a necessity that must be carried out by the community even in the situation of the coronavirus disease-19 (Covid-19) pandemic, which is not yet known when it will end. This can be understood, because sport for the community has the function of obtaining physical and mental health. By doing sports activities, it will indirectly prevent a disease from coming. Likewise with the benefits for mental health, where when we do sports activities, the body will release endorphins which function as a trigger for feelings of pleasure and happiness. This hormone can reduce the bad effects of feelings of stress and pain, increase appetite and immune response.

Some of the functions of sports activities to obtain physical health, as stated by (Lumb, 2014) exercise have a beneficial effect on metabolic parameters that affect cardiovascular risk, such as lipids, blood glucose, and are a key component in the prevention and management of type 2 diabetes. While Kruk, (2009) stated that physical activity is an important determinant of physical and psychological health. Regular physical activity has a beneficial effect on the onset and development of several chronic diseases, well-being, and has a positive effect on the community and society. Unfortunately, more than $60 \%$ of adults worldwide do not reach the recommended level of physical activity. Likewise, according to Okafor, C. I., Gezawa, I. D., Sabir, A. A., et al., (2013) the benefits of physical activity are very broad and go beyond health alone. Being physically active is a major contributor to a person's overall physical and mental well-being. Positive results include a better quality of life, improved sleep, and less stress, as well as stronger relationships in social interactions.

In a WHO statement, (2018a) that regular physical activity aims to prevent and treat major non-communicable diseases (PTM), namely heart disease, stroke, diabetes, and breast and colon cancer. It also contributes to the prevention of other important NCD risk factors such as hypertension, being overweight and obesity, and is associated with improved mental health, delayed onset of dementia and improved quality of life and well-being. In line with that, Owen et al., (2010) stated that when adults meet physical activity guidelines, sitting for long periods of time can interfere with metabolic health. Sitting time, watching TV time, and sitting in the car increase the risk of premature death. Furthermore, Simon J. Marshall, PhD, and Ernesto Ramirez, MS, (2011) stated that studies of sedentary behavior and health outcomes (phase I) have found consistent evidence that excessive use of screen-based media is associated with overweight and obesity in children, and there is some evidence among adults that overall sedentary time is associated with cardiometabolic risk factors, some diseases, cancer, and death. 


\section{b. Recreation}

Sports activities will be recreational in nature if they are carried out at low intensity and in a more relaxed state. Although low intensity is basically for everyone one another is not the same. Therefore, the initial physical fitness must be known so that a person can determine what the low intensity is for himself. The community should be able to find out the initial conditions, especially cardiovascular fitness by taking simple measurements, for example doing step tests, running $2.4 \mathrm{~km}$, multistage (running back and forth) and so on. However, if this initial fitness is not known yet, then continue to do sports activities gradually. Do it starting from light movements and short time. No need to force yourself to start with the movements that each individual likes best.

\section{c. Social Interaction}

Social interaction can be done by doing sports activities together both formally and informally. Formal, which is meant if sports activities are carried out in schools, agencies, and other formal institutions. Meanwhile, the informal ones are sports activities carried out by the community which are carried out on their own initiative and form a sports community. As social beings, community sports activities are still needed so that social needs can be met. So, in this context sports activities as a tool to meet the needs of individuals as members of society. New normal is a necessity that must be passed by people in almost all over the world. Therefore, what is possible is to do sports activities as usual before the pandemic, but by applying selfdiscipline to health protocols by maintaining distance, not crowding, wearing masks, washing hands with soap/hand sanitizer.

\section{OPTIONAL TYPE OF SPORTS}

It is necessary, the authors provide an overview of several types of sports that can be done individually or in groups. In the context of the COVID-19 pandemic, the authors recommend that people prefer individual types of sports, but if forced to keep exercising outside or in public places, choose sports that do not occur in direct physical contact. Below are some of the groupings of sports as follows.

\section{Individual Sports}

Quite a few types of sports are individual, but there are several examples of sports that are individual and are often seen or known by many people, including: surfing, swimming, diving, badminton, table tennis, tennis, gymnastics, athletics, archery, cycling, wrestling, boxing, martial arts, mountaineering, bowling, golf, and so on.

\section{Team Sports}

Meanwhile, there are also quite a lot of team sports, including baseball, basketball, field hockey, soccer, volleyball, futsal, handball, ice hockey, indoor and outdoor handball, water polo, and so on.

\section{NEW NORMAL AT THE PANDEMI CORONAVIRUS} DISEASE-19

\section{Meaning New Normal}

If countries in the world have not found a cure or vaccine for the treatment and cure of COVID-19, we may have to adjust to the "new normal", which means a new way to live and live our lives, work, and our interactions with other people (Media, n.d). Wiku Adisasmito, Chair of the Expert Team for the Task Force for the Acceleration of Handling of COVID-19, said that new normal is a change in behavior to continue carrying out normal activities but with the addition of implementing health protocols to prevent transmission of COVID-19 (Know the Term The New Normal, n.d.). According to Yuri, new orders, habits, and behaviors based on adaptation to cultivate a clean and healthy lifestyle are then called new normal (Meaning New Normal Indonesia, n.d.).

\section{New Normal Principle}

Basically, the principle of this new normal is how people continue to carry out their daily activities like before the pandemic but must adapt to health protocols as an effort to break the chain of spreading COVID-19. These health protocols include, 1) the obligation to use a mask when outside the house, 2) maintain a social distance of at least 1 (one) meter when carrying out various activities, 3) the habit of washing hands with soap or hand sanitizer before or after activities, 4) measuring body temperature, 5) avoiding direct contact, such as shaking hands.

\section{SPORT ACTIVITIES WITH FITT PRINCIPLES WHEN NEW NORMAL}

\section{Benefits of Sports Activities Using the FITT Principles}

FITT is an acronym for frequency, intensity, time, and type. FITT can be used to make it easier for people to remember and carry out sports activities by referring to this acronym. As an illustration, 1) frequency, can be defined by how many times a week the exercise is done, 2) intensity, means how heavy the exercise activity is carried out. This intensity category includes light, moderate and heavy intensity, 3) time, which means how long the sports activity is carried out. Usually measured in minutes, 4) types are the types of sports that can be selected, such as individual or team sports.

The FITT program significantly contributes to the improvement of aerobic fitness, muscle fitness and flexibility. The FITT intervention is a promising school strategy to improve a student's aerobic and muscular fitness (Cvejic \& Ostojić, 2018a). Furthermore, Casebolt, (2009) states that increased knowledge of health-related fitness concepts such as fitness assessment, goal setting, and application of the FITT principle 
(frequency, intensity, time, and type) will result in increased activity. Likewise, according to Ferkel et al., (2019) the truth of the movement of health-related fitness education can empower students to create and implement a lifelong fitness plan through learning skills (for example, the possibility of exercise) and knowledge (for example, goal setting, FITT principles, planning) necessary to stay in shape. Health-related fitness education also helps develop self-efficacy and other affective attributes that can motivate lifelong adherence.

In another study Burnet et al., (2019) stated that the prevalence of physical inactivity continues to increase even though there is sufficient evidence to show the right dose of exercise to reduce the risk of many non-communicable diseases. This increase in physical activity may not be related to knowledge of exercise prescriptions, but rather to the way of exercise. Current exercise prescription guidelines are characterized by the FITT Principles: Frequency, Intensity, Time, and Type. This is almost the same as stated by Oberg, (2007) the principles of FITT - frequency, intensity, time, and type of exercise - can be used to adjust physical activity recommendations to the needs and goals of each patient. Frequency should be prioritized when health goals are related to chronic disease prevention. Intensity has the maximum impact on weight loss and athletic conditioning. Timing is very relevant for people with diabetes and blood sugar dysregulation. Types of activity include aerobic, balance, flexibility, and strength exercises, all of which help patients achieve different health goals. Furthermore, according to Barisic et al., (2011) physical activity is a multi-faceted behavior consisting of several components: frequency, intensity, time, and type (FITT). There is currently a lack of individual knowledge about how the components of FITT affect chronic disease. This is partly due to the tendency for researchers to focus on intensity through accurate measurement of energy expenditure. Although energy expenditure is an important consideration in assessing physical activity, it is only one component. Thus, future studies examining the association between physical activity and disease risk would benefit from examining all components of FITT.

According to Katsukawa, (2016) the principle of FITT training is a framework for training recipes. A general recommendation is for at least 30 minutes of moderate intensity exercise on most days of the week (150 minutes total per week). In this article, the evidence is reviewed to clarify the optimal exercise conditions for managing lifestyle-related diseases such as hypertension, diabetes, dyslipidemia, obesity, and metabolic syndrome.

\section{Benefits of Sports Activities in General}

From several research results on the benefits of physical activity on physical and mental health, there are quite a lot of similar studies that support it. The results, mostly indicate that physical activity that is emphasized on sports activities has a positive impact, such as the research below.

According to Kutluturk et al., (2017) the level of student physical activity increases, the percentage of body fat decreases, flexibility, muscle endurance and aerobic fitness increase. To prevent the adverse effects of inactivity on physical fitness, it is thought that students should be encouraged to engage in more physical activity. Similar thing by WHO, (2018b) physical inactivity is the fourth cause of death in the world. We summarize current global efforts to address this problem and point the way forward to tackling the pandemic of physical inactivity. Likewise, according to Perez-Terzic, (2012) extensive data analysis has shown that sports training has a significant impact on the prevention and modification of cardiovascular disease and mortality. In general, exercise recommendations for patients with cardiovascular disease are based on individual aerobic capacity and comorbidities.

In their research, Zisser et al., (2011) stated that diet and exercise form the foundation of a healthy lifestyle. This is especially important for people living with diabetes mellitus, as they are the most practical non-pharmacological means by which patients can significantly increase their blood glucose levels. Exercise increases insulin sensitivity (both short and long term), lowers blood sugar levels, reduces body fat, and improves cardiovascular function (CV). Furthermore, De Rezende et al., (2015) stated that in Brazil, public policies and interventions that promote physical activity will significantly improve population health. Physical activity has a major impact on NCD and mortality, especially in the southeast and central-west regions. Public policies and interventions that promote physical activity will significantly improve population health.

The results of the research Power \& Clifford, (2013) exercising for at least 1 hour/week for at least 40 hours for the required intervention, succeeded in reducing the incidence of falls. The optimal training frequency is three times per week, but the optimal duration per exercise is still unclear. Specific balance training of sufficiently challenging intensity is a vital component of a program, and strength training is most effective when combined with balance training. Flexibility and resistance training can also be included as part of a comprehensive program. A combination of group and individual home exercises may be most effective at preventing falls and improving exercise compliance. Furthermore, Bulger, (2011) states that workrelated physical activity, in terms of peak loads and continuous and/or repetitive contractions, presents a risk factor for the development of pain and muscle disorders. However, physical activity as training that is tailored to the work exposure, health, and physical capacity of employees offers prevention and rehabilitation. We suggest the concept of "Intelligent Physical Exercise" relies on evidence-based sports science training principles. 
The results of the study by Liao et al., (2017) reveal: (1) the basic functional movement ability of young people is low, and correction training is needed for them to move well first before improving physical fitness performance, (2) low correlation between Functional Movements Screen (FMS) and Physical Fitness Test (PFT) show that movement quality and physical performance are two independent abilities and cannot be replaced by one another. Similar points were stated by Riiser et al., (2014) that internet intervention with tailored physical activity counseling can have short-term beneficial effects on cardiorespiratory fitness, Health-Related Quality of Life (HRQoL) and Body Mass Index (BMI). among overweight and obese adolescents. Likewise, according to Chen, (2016) that children should regularly participate in exercise, because fitness can improve the development of peer relationships and selfesteem. In addition, fitness instructors can provide participants with a suitable level or gradually increase fitness intensity in the principles of fitness that can improve and build children's peer relationships.

According to the study of O'Riordan et al., (2013) physiotherapy intervention using a multimodal approach appears to produce more favorable outcomes in terms of increased strength, improved function, and quality of life associated with health and reduced pain scores. Active strengthening exercises appear to be beneficial for all of these results; the inclusion of additional stretching and aerobic exercise components appears to enhance the benefits of exercise interventions. Likewise, according to Fiatarone Singh \& Morley, (2012) exercise is the most effective approach to delaying the aging process. It also reverses sarcopenia, weakness and disability. Different types of exercise include aerobics, resistance (strength), balance, flexibility and posture. Exercise is the key to an active lifestyle. Dipla, (2017) states that sport is a recipe that must be tailored to individual needs to achieve maximum benefits and high compliance.

In their research, Rivera-Brown \& Frontera, (2012) stated that physical activity and fitness are associated with a lower prevalence of chronic diseases, such as heart disease, cancer, high blood pressure, and diabetes. This review examines the body's response to acute bouts of exercise and long-term physiological adaptations with an emphasis on resistance training. According to $\mathrm{Wu}$ et al., (2016) fitness trackers have expanded the health care ecosystem and made it possible for daily physical activities to be carried out alone. Features such as heart rate monitoring can help detect health ailments, but there is little evidence to suggest tracking health and physical activity indicators that lead to long-term changes in health behavior.

In Katzmarzyk's research, (2016) stated that life expectancy can be increased by more than 10 months if Canadians can be encouraged to be physically active. The same thing was stated by Waleh, (2016) about two-thirds of the US population is overweight or obese. Physical activity is recommended to prevent obesity, aid in weight loss, and reduce rates of chronic disease. Furthermore, Lee et al., (2020) stated that behavior-based exercise interventions, use of behavior change strategies, supervision by experienced fitness instructors, and fun sports can improve exercise and adherence to a specified exercise program.

In their research, Ayers \& Sariscsany, (2010) stated that the best strategy for physical education teachers is to include fitness and health-related activities into physical education programs. Teachers learn to develop curricula based on current national standards and guidelines, apply the concept of fitness in real-world settings, and motivate students to live healthy, active lives. Furthermore Ward et al., (2017) in their research that configuring the main principles of exercise education in a fitness unit is an efficient model to provide students with the opportunity to improve fitness skills and knowledge (HRF) while achieving moderate to heavy physical activity levels (MVPA). recommended. Likewise, according to Gray et al., (2015) the development of motor skills is not related to time outside; However, this association was only examined in a single study of preschool children. No studies have found an association between time outdoors and musculoskeletal fitness.

In this paper, the authors wish to emphasize that doing sports activities has excellent physical and mental health benefits. However, the authors advise the public to continue doing sports activities guided by the principles of FITT training and following the COVID-19 protocol. The advantage of this principle is that it makes it easier for us to measure how to do sports properly and is also easy to serve as a guide. This FITT principle can be carried out in various ways, depending on the ability of everyone who performs it. For example, the principle of time (time), each may do as he wishes, whether 15,20 , or 25 minutes. This depends on the initial fitness of each. Including the type (type) of sports, each can choose the sport or physical activity that is most interested and preferred.

\section{CONCLUSION}

Moving is a basic human need to fulfill their daily needs by working, caring for and maintaining oneself to stay healthy. Therefore, people still must do physical activity/sports to keep themselves healthy and fit, as well as improve their motor function to fulfill their basic needs.

The benefits that can be obtained by doing physical activity/sports include: 1) physical and mental health, if a person has a good level of physical fitness, then physically he will not tire easily after carrying out activities in fulfilling his daily duties, 2) exercise is done as a the main objective for recreational activities, meaning that in addition to physical health benefits, 
the main objective is actually being healthy from a mental aspect, 3) as well as being used as a tool for social interaction with various colleagues in their respective environments.

The definition of "New normal" is basically carrying out activities of daily life such as before the COVID-19 pandemic attacked mankind, but accompanied by implementing health protocols. Meanwhile, the principle of "New normal" is an activity of daily life normally, at the same time following health protocols, among others, 1) the obligation to use a mask when outside the home, 2) maintain a social distance of at least 1 (one) meter when carrying out various activities, 3 ) the habit of washing hands with soap or hand sanitizer before and after activities, 4) measuring body temperature, 5) avoiding direct contact, such as shaking hands.

The principles of FITT training are basically a guideline to make it easier for people who do physical activities/sports. Frequency, defined how many times a week do sports activities. Here, the authors recommend at least 3 times a week. Intensity, defined as the severity of a physical activity/sport. For beginners, you should start with light intensity, and if you feel fitter you can go to moderate intensity. Mild intensity if the pulse is below $60 \%$ of the maximum pulse rate $(220$-age $) \times 80 \%=160$. This means that if we determine $60 \%$ of the exercise, our pulse will be around 96 per minute.

The benefits of doing physical activity / sports are between those using the FITT guidelines and those that are not basically the same, both of which can improve physical and mental fitness. However, based on the principles of this FITT exercise, the community has a clearer direction in their activities. By using the FITT guidelines, physical fitness will improve and simultaneously contribute to the management of several diseases related to lifestyle such as diabetes, hypertension, dyslipidemia, obesity, and metabolic syndrome. In addition, from the mental health aspect, we can develop self-efficacy and other affective attributes that can motivate lifelong adherence.

\section{REFERENCES}

Arti New Normal Indonesia: Tatanan Baru Beradaptasi dengan COVID-19. (n.d.). tirto.id. Retrieved June 29, 2020, from https://tirto.id/arti-new-normal-indonesiatatanan-baru-beradaptasi-dengan-covid-19-fDB3.

Ayers, S. F., \& Sariscsany, M. J. (2010). FITT. In the National Association for Sport and Physical Education.

Barisic, A., Leatherdale, S. T., \& Kreiger, N. (2011). Importance of frequency, intensity, time, and type (FITT) in physical activity assessment for epidemiological research. Canadian Journal of Public Health. https://doi.org/10.1007/bf03404889

Bulger, S. (2011). Basic Training Principles. Physical Education for Lifelong Fitness.

Burnet, K., Kelsch, E., Zieff, G., Moore, J. B., \& Stoner, L. (2019). How fitting is F.I.T.T.? A perspective on a transition from the sole use of frequency, intensity, time, and type in exercise prescription. In Physiology and

Behavior https://doi.org/10.1016/j.physbeh.2018.11.007

Casebolt, K. (2009). Physical Activity in Higher Education. Pennsylvania Journal of Health, Physical Education, Recreation \& Dance.

Chen, H. C. (2016). The Impact of Children's Physical Fitness on Peer Relations and Self-Esteem in School Settings. Child Indicators Research https://doi.org/10.1007/s12187-015-9330-6

Cvejic, D., \& Ostojić, S. (2018a). Effects Of The Fitt Program On Physical Activity And Health-Related Fitness In Primary School Age Children. Facta Universitatis, Series: Physical Education and Sport. https://doi.org/10.22190/fupes1703437c

Cvejic, D., \& Ostojić, S. (2018b). Effects of the Fitt Program on Physical Activity and Health-Related Fitness in Primary School Age Children. Facta Universitatis, Series: Physical Education and Sport, 15(3), 437. https://doi.org/10.22190/fupes1703437c

De Rezende, L. F. M., Rabacow, F. M., Viscondi, J. Y. K., Luiz, O. D. C., Matsudo, V. K. R., \& Lee, I. M. (2015). Effect of physical inactivity on major noncommunicable diseases and life expectancy in Brazil. Journal of Physical Activity and Health. https://doi.org/10.1123/jpah.2013-0241

Dipla, K. (2017). The FITT Principle in Individuals with Type 2 Diabetes: From Cellular Adaptations to Individualized Exercise Prescription. Journal of Advances in Medicine and Medical Research. https://doi.org/10.9734/jammr/2017/34927

Ferkel, R. C., Hutchinson, Z. T., Razon, S., True, L., Zupin, D., Jones, L. M., \& Judge, L. W. (2019). The Benefits of Health-Related Fitness Education in Secondary PE. The Physical Educator. https://doi.org/10.18666/tpe2019-v76-i4-8724

Fiatarone Singh, M. A., \& Morley, J. E. (2012). Physical Fitness and Exercise. In Pathy's Principles and Practice of Geriatric Medicine: Fifth Edition. https://doi.org/10.1002/9781119952930.ch10

Gray, C., Gibbons, R., Larouche, R., Sandseter, E. B. H., Bienenstock, A., Brussoni, M., Chabot, G., Herrington, S., Janssen, I., Pickett, W., Power, M., Stanger, N., Sampson, M., \& Tremblay, M. S. (2015). What is the relationship between outdoor time and physical activity, sedentary behaviour, and physical fitness in children? A systematic review. In International Journal of Environmental Research and Public Health. https://doi.org/10.3390/ijerph120606455

Katsukawa, F. (2016). FITT principle of exercise in the management of lifestyle-related diseases. In Clinical calcium. https://doi.org/CliCa1603447451

Katzmarzyk, P. T. (2016). Physical Inactivity and Life Expectancy in Canada. Journal of Physical Activity and Health. https://doi.org/10.1123/jpah.3.4.381 
Kruk, J. (2009). Physical activity and health. Asian Pacific Journal of Cancer Prevention. https://doi.org/10.4324/9781351217385-3

Kutluturk, S., Aras, G., Ergezen, G., \& Mutluay, F. (2017). Investigation of physical activity level and physical fitness of university students. Fizyoterapi Rehabilitasyon.

Lee, H. S., Lee, S. H., Kim, J. W., Lee, Y. S., Lee, B. C., Oh, H. J., \& Kim, J. H. (2020). Development of Novel Continuous and Interval Exercise Programs by Applying the FITT-VP Principle in Dogs. Scientific World Journal. https://doi.org/10.1155/2020/3029591

Liao, T., Zheng, W., \& Meng, Y. (2017). Application of functional movement screen to the evaluation of youth's physical health. Advances in Intelligent Systems and Computing. https://doi.org/10.1007/9783-319-41953-4_17

Lumb, A. (2014). Diabetes and exercise. Clinical Medicine, Journal of the Royal College of Physicians of London. https://doi.org/10.7861/clinmedicine.14-6-673

Media, K. C. (n.d.). Mengenal Apa Itu New Normal di Tengah Pandemi Corona... Halaman all. KOMPAS.com. Retrieved June 29, 2020, from https://www.kompas.com/tren/read/2020/05/20/06310 0865/mengenal-apa-itu-new-normal-di-tengahpandemi-corona-

Mengenal Istilah The New Normal: Sebuah Kondisi Karena Pandemi. (n.d.). Mengenal Istilah The New Normal: Sebuah Kondisi Karena Pandemi. Retrieved June 30, 2020, from https://www.enervon.co.id/article/1047/mengenalistilah-the-new-normal-sebuah-kondisi-karenapandemi/.

Oberg, E. (2007). Physical activity prescription: Our best medicine. In Integrative Medicine.

Okafor, C. I., Gezawa, I. D., Sabir, A. A., Raimi, T. H., Enang, O., Geisler, C., ... Senarath, U. (2013). Health Promotion Model - Instruments to Measure HPM Behavioral Determinants : Exercise Social Support Scale (Adolescent Version). The Lancet. https://doi.org/10.7600/jpfsm.1.325

O'Riordan, C., Clifford, A., Van De Ven, P., \& Nelson, J. (2013). Chronic Neck Pain \& Exercise Interventions: The FITT principle. In Archives of physical medicine and https://doi.org/10.1016/j.apmr.2013.11.015

Owen, N., Healy, G. N., Matthews, C. E., \& Dunstan, D. W. (2010). Too Much Sitting. Exercise and Sport Sciences Reviews

https://doi.org/10.1097/jes.0b013e3181e373a2
Perez-Terzic, C. M. (2012). Exercise in cardiovascular diseases. $P M$ and $R$. https://doi.org/10.1016/j.pmrj.2012.10.003

Power, V., \& Clifford, A. M. (2013). Characteristics of optimum falls prevention exercise programmes for communitydwelling older adults using the FITT principle. In European Review of Aging and Physical Activity. https://doi.org/10.1007/s11556-012-0108-2

Riiser, K., Løndal, K., Ommundsen, Y., Småstuen, M. C., Misvær, N., \& Helseth, S. (2014). The outcomes of a 12-week internet intervention aimed at improving fitness and health-related quality of life in overweight adolescents: The young \& active controlled trial. PLoS ONE. https://doi.org/10.1371/journal.pone.0114732

Rivera-Brown, A. M., \& Frontera, W. R. (2012). Principles of exercise physiology: Responses to acute exercise and long-term adaptations to training. $P M$ and $R$. https://doi.org/10.1016/j.pmrj.2012.10.007

Simon J. Marshall, PhD, dan Ernesto Ramirez, MS, 2011 . (2011, November 5). Reducing Sedentary Behavior: A New Paradigm in Physical Activity Promotion. ResearchGate.

https://www.researchgate.net/publication/254075466_ Reducing_Sedentary_Behavior_A_New_Paradigm_in _Physical_Activity_Promotion

Waleh, M. Q. (2016). Impacts of Physical Activity on the Obese. In Primary Care-Clinics in Office Practice. https://doi.org/10.1016/j.pop.2015.08.014

Ward, J. K., Hastie, P. A., Wadsworth, D. D., Foote, S., Brock, S. J., \& Hollett, N. (2017). A Sport Education Fitness Season's Impact on Students' Fitness Levels, Knowledge, and In-Class Physical Activity. Research Quarterly for Exercise and Sport. https://doi.org/10.1080/02701367.2017.1321100

WHO? (2018a). Global action plan on physical activity 2018-2030: More active people for a healthier world. Geneva: World Health Organization; 2018. Licence: CC BY-NC-SA 3.0 IG. In Who. https://doi.org/10.1016/j.jpolmod.2006.06.007

WHO. (2018b). Global action plan on physical activity 2018 2030: More active people for a healthier world. Geneva: World Health Organization; 2018. Licence: CC BY-NC-SA 3.0 IG. In Who. https://doi.org/10.1016/j.jpolmod.2006.06.007

Wu, Q., Sum, K., \& Nathan-Roberts, D. (2016). How Fitness trackers facilitate health behavior change. Proceedings of the Human Factors and Ergonomics Society. https://doi.org/10.1177/1541931213601247

Zisser, H., Gong, P., Kelley, C. M., Seidman, J. S., \& Riddell, M. C. (2011). Exercise and diabetes. In International journal of clinical practice. Supplement. https://doi.org/10.1111/j.1742-1241.2010.02581.x 